Am J Gastroenterol. 2000 Sep;95(9):2301-7.

\title{
High frequencies of telomeric associations, chromosome aberrations, and sister chromatid exchanges in ulcerative colitis.
}

Cottliar A, Fundia A, Boerr L, Sambuelli A, Negreira S, Gil A, Gómez JC, Chopita N, Bernedo A, Slavutsky I.

Departamento de Genética, Instituto de Investigaciones Hematológicas Mariano R Castex, Academia Nacional de Medicina, Buenos Aires, Argentina.

\section{Abstract}

OBJECTIVE: Chromosome instability provides a predisposing background to malignancy, contributing to the crucial genetic changes in multistep carcinogenesis. The aim of this work was to analyze chromosome instability in patients with ulcerative colitis (UC) to achieve a better understanding of the increased risk for colorectal cancer. METHODS: Peripheral blood lymphocyte cultures from 20 untreated UC patients and 24 controls were used to study chromosome instability by assessing telomeric associations (TAS), chromosome aberrations (CA), and sister chromatid exchanges (SCE). RESULTS: Mean frequencies of TAS and CA were significantly increased in UC patients compared to controls ( $p<0.001)$. Chromosomes $10,11,21,16$, and 19 were the most frequently involved in TAS. A total of 104 CA clustered in 66 breakpoints could be exactly localized. Seven nonrandom bands significantly affected in UC patients were found $(p<0.004)$, showing a significant correlation with the location of cancer breakpoints $(p<0.003)$, particularly with colorectal carcinoma rearrangements. SCE analysis showed higher levels in patients compared to controls $(p<0.006)$, but no differences were observed in cell cycle kinetics. CONCLUSIONS: Our results demonstrate the presence of an unstable genome in UC patients that could be related to the cancer development observed in this disease. 